\title{
Minimally invasive surgery for pneumothorax: the evidence, changing practice and current opinion
}

Tom Treasure

J R Soc Med 2007; 100:419-422

\section{SUMMARY}

Spontaneous pneumothorax is a common problem and in patients at risk of recurrence, excision of the source of the air leak and pleurodesis is very effective in reducing further episodes. In the 15 years since the introduction of video assisted thoracic surgery (VATS), practice has changed towards this much less invasive procedure and away from thoracotomy. The evidence from randomized trials, nonrandomized comparative studies, the consensus of surgeons and the documented change in practice from registry data are concordant. Unless there are specific clinical indications for more invasive surgery, it would seem reasonable for VATS to become the recommended approach.

\section{BACKGROUND AND INTRODUCTION}

Video assisted thoracic surgery (VATS) developed from laparoscopic surgery; it was first described in the surgical literature in 1992.1,2 Within ten years, VATS had been widely adopted for lung and pleural diagnostic procedures (e.g. biopsy) and was increasingly used for the management of pneumothorax. The thorax lends itself to the VATS approach more readily than does the abdomen: it has been said that with the lung on the operated side collapsed and the chest wall supported by arches of the ribs 'it's like operating in a cathedral'.

In this review I summarize:

- Existing guidance on pneumothorax surgery;

- Existing evidence for the choice between VATS and thoracotomy;

- Changing practice in the UK over time;

- A formal opinion gathering exercise amongst UK thoracic surgeons.

\section{EXISTING GUIDANCE}

The indications for and objectives of pneumothorax surgery are set out in guidelines from the British Thoracic Society (BTS). ${ }^{3}$ A best estimate is that while after a first pneumothorax less than a third of patients experience recurrence, after three or more episodes most patients

Professor of Cardiothoracic Surgery, Guy's Hospital, London

Email: Tom.Treasure@gmail.com
(60-70\%) will have another pneumothorax. Elective surgery for pneumothorax is therefore offered after two or more episodes in the belief that this defines a group in whom recurrence is much more likely than not. The objective of elective surgical intervention is to prevent recurrence by removing - as far as technically feasible - the causative blebs and bullae at the apex of the lung and to create a pleurodesis so that any future leaks cannot progress to pneumothorax. There is no practical way of checking if pleural symphysis has been achieved, so to denote failure we rely on clinically evident recurrence of pneumothorax, confirmed on chest X-ray.

All reported techniques bring the recurrence rate down to low single figures. The BTS guidelines refer to thoracotomy and surgical pleurectomy as the 'gold standard', with a purported recurrence rate of less than $1 \%$. The BTS guidelines state that VATS must be evaluated against this gold standard.

\section{THE EVIDENCE BASE}

There is an evidence base for the choice between VATS and thoracotomy. In 2004, Sedrakyan et al. published a systematic review of the evidence, covering four RCTs directly comparing the outcomes between VATS and thoracotomy for pneumothorax, and incorporating a total of 90 VATS procedures and 89 thoracotomies. ${ }^{4}$ Eleven failures $(6 \%)$ were recorded during the follow up time, but there were too few failures in any one study to show significant differences between the techniques. This failure rate is far higher than that in the retrospective series cited in the BTS guidelines. It should be noted that a trial powered on the basis of these data to show a difference in failure rates of, say, $8 \%$ to $4 \%$ at this level would require nearly 1000 patients.

A second outcome measure for these procedures is the achieved reduction in pain and physical limitation. In terms of the systematic review by Sedrakyan et al., how well does VATS deliver the reduction in pain and physical limitation that is hoped for, compared to thoracotomy? ${ }^{4}$ All four RCTs reported reduced analgesic requirements and decreased length of hospital stay for the VATS group. This result raises an important methodological question: the RCTs were reported between 1994 and 2000 by surgeons 
who were early adopters of VATS, and who might well have been seeking to prove its advantages. Pain and its management are subjective and negotiated, and as for length of stay- 'when the professor says you can go home, you go home'. Another consideration is that, while the new technique might succeed in the hands of the practised VATS enthusiast, in the hands of a surgeon not skilled in VATS, patients may be better off having a thoracotomy.

In some clinical circumstances where the treatment and the practitioner are not easily separated (as in psychotherapy as well as surgery) an expertise-based study may be appropriate. ${ }^{5}$ Such a study occurred by chance when a younger surgeon adopted VATS, while the senior surgeon continued to use a thoracotomy. ${ }^{6}$ Both surgeons were using the technique they preferred and with which they were practised and confident. The retrospective nature of the study can be turned to advantage: the analgesic usage and pain clinic information was as clinically indicated at the time, uncontaminated by the knowledge that it would later be used as an outcome in judging between two procedures. The difference in opiates taken at one, two and four weeks was highly significant. Referral to the pain clinic was $13 \%$ $(8 / 63$; $95 \%$ confidence limits $5-22 \%)$ in the thoracotomy group; there were no referrals to the pain clinic from the VATS group.

\section{CHANGING PRACTICE}

An analysis of the Thoracic Surgical Register of the Society of Cardiothoracic Surgeons of Great Britain and Ireland (SCTS) for two years from 2000 to 2002 revealed that $57 \%$ of 2606 operations for pneumothorax were performed thoracoscopically. ${ }^{7}$ Practice varied widely, from $0-100 \%$ across the forty hospitals reporting (Figure 1). This variation was independent of the number of pneumothorax operations performed and did not correlate with the use of VATS for other procedures. It appeared to be a matter of the surgeons' choice.

The UK data for the years 2003, 2004 and 2005 (Table 1) show a change in practice compared with the data reported for 2000-2002,7 with the proportion of operations performed by VATS increasing from $57 \%$ to over $70 \%$.

Are these registry data reliable? They are voluntary returns of members of the SCTS, and not all units returned their data every year, so the absolute numbers are, in all likelihood, undercounts. However, the data provided in the 2000-2002 analysis were from years with high returns and they showed no correlation between the proportion of pneumothorax operations performed by VATS and the total number of cases or other measures of VATS practice. The numbers are large, the condition is well defined and the procedures are amenable to counting. It is therefore

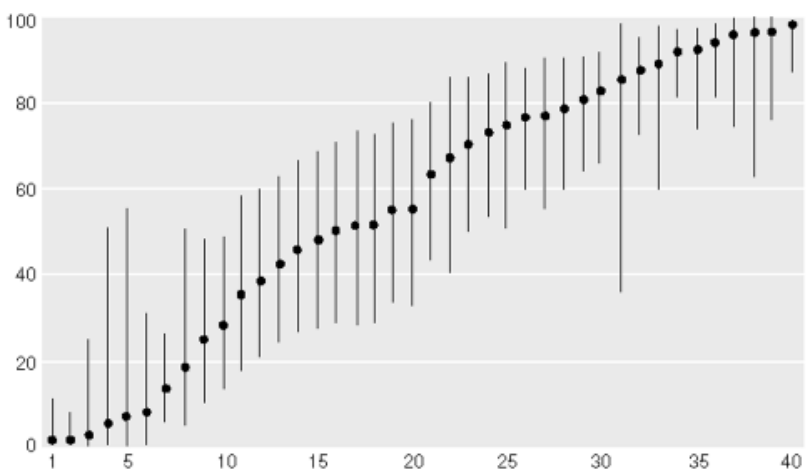

Figure 1 Proportion of thoracotomies and VATS performed in forty hospitals.

possible to conclude that there probably has been a real and substantial change in practice towards adoption of VATS.

\section{AN OPINION SEEKING EXERCISE}

A meeting of the Cardiothoracic Section of the Royal Society of Medicine (Friday 17 November 2006) provided an opportunity to sample the opinions of a wide range of cardiothoracic surgeons. I presented the key data from the 2000-2002 analysis to the meeting, showing that there was a wide range of practice at the time, but no interpretation or comment was made on the appropriateness or otherwise of this practice (Figure 1).

I then presented the audience with three statements which I believed captured the flavour of the opposing opinions commonly expressed in support of one or other method:

(1) The optimal operation for pneumothorax is still through a thoracotomy;

(2) Surgery for pneumothorax is now more easily achieved by VATS;

(3) Using 'my' thoracotomy technique (that is, the technique of the responding surgeon), there is no advantage to the patient in having VATS.

Table 1 Numbers of thoracotomies and VATS procedures performed by year

\begin{tabular}{lllllll}
\hline \multirow{2}{*}{ Year } & \multicolumn{2}{l}{ Thoracotomy } & & \multicolumn{2}{l}{ VATS } & \multicolumn{2}{c}{ Total } \\
& $\boldsymbol{n}$ & $\%$ & $\boldsymbol{n}$ & $\%$ & \\
\hline 2003 & 372 & $30 \%$ & 872 & $70 \%$ & 1244 \\
2004 & 386 & $26 \%$ & 1077 & $74 \%$ & 1463 \\
2005 & 460 & $27 \%$ & 1234 & $73 \%$ & 1694 \\
\hline
\end{tabular}




\begin{tabular}{|c|c|c|c|c|c|c|c|}
\hline & $\begin{array}{l}\text { Agree } \\
\text { strongly }\end{array}$ & Agree & Disagree & $\begin{array}{l}\text { Disagree } \\
\text { strongly }\end{array}$ & Total votes & $\%$ For & $\%$ Against \\
\hline $\begin{array}{l}\text { The optimal operation for } \\
\text { pneumothorax is still through a } \\
\text { thoracotomy }\end{array}$ & 4 & 7 & 26 & 18 & 55 & $20 \%$ & $80 \%$ \\
\hline $\begin{array}{l}\text { Surgery for pneumothorax is now } \\
\text { more easily achieved by VATS }\end{array}$ & 25 & 20 & 6 & 1 & 52 & $87 \%$ & $13 \%$ \\
\hline $\begin{array}{l}\text { Using my technique of thoracotomy } \\
\text { there is no advantage to the patient } \\
\text { in having VATS }\end{array}$ & 1 & 3 & 34 & 15 & 53 & $8 \%$ & $92 \%$ \\
\hline
\end{tabular}

In two further questions I asked which method the surgeons themselves would prefer if a relative were to undergo pneumothorax surgery. To limit the inevitable 'it all depends' prevarication, the question was narrowed to a male aged 15-30 with the assumption that all operations would be performed 'in good hands' by surgeons practised in the technique.

The question was first put for an elective operationthat is, a planned interval procedure in a patient who had two or more episodes of primary spontaneous pneumothorax. The same question was then put for a 'rescue' operation. This was explained as any situation in which the operation was deemed necessary on that admission, rather than an absolute emergency. Examples include ongoing air leak, failure of the lung to re-expand, bleeding, or the onset of infection.

All the questions were presented for the participants' consideration and definitions were explained before any voting was done. The voting was by electronic touch pads. Only after all the votes were cast were the answers revealed.

Most of the surgeons present $(80 \%)$ did not believe that thoractomy was required for an optimal operation - in contradiction to the extant guidelines - and $87 \%$ believe it is more easily done by VATS (Table 2). If their own family member were to have an elective operation, $79 \%$ would prefer VATS to be used; however, in a non-elective situation the largest vote (44\%) was to leave the choice of technique to the surgeon (Table 3 ).

\section{DISCUSSION}

I am well aware of the limitations and potential flaws in using what are generally known as 'consensus methods' ${ }^{8}$ Limitations in this exercise include the fact the opinions were only of those present. The day was devoted to various aspects of minimally invasive surgery of the heart and lungs, so it is possible that there was a bias towards VATS adopters - it is likely that at any meeting there are a disproportionate number of opinion leaders. On the other hand, there have been strong opinions against VATS in the past; the voting was secret and remained anonymous; and the participants represented the rank and file of British cardiothoracic surgeons and their trainees, so we can reasonably accept the result as indicative of the opinions of cardiothoracic surgeons.

The first two questions put to the participants were loaded in opposite directions, but in whichever direction the questions were spun the results showed a very large majority in favour of VATS as a satisfactory way of achieving the objectives of surgery. The third question moved the emphasis to the patient's experience. There is an argument used in mitigation for not adopting the new technique that a traditional full thoracotomy is not a fair comparison-a smaller, muscle sparing incision may achieve the same benefits for the patient. The overwhelming majority of those present at the meeting (92\%) do not now hold that view. When it came to a scenario where a young male relative was to be operated upon, the

Table 3 Surgeon's preferences for thoracotomy or VATS in elective and 'rescue' sitations

\begin{tabular}{llll}
\hline Scenario of a 15- to 30 -year old male relative & Thoracotomy & VATS & Leave it entirely to the surgeon \\
\hline Elective & $2 \%$ & $79 \%$ & $19 \%$ \\
'Rescue' & $14 \%$ & $42 \%$ & $44 \%$ \\
\hline
\end{tabular}


majority of surgeons wanted them to have VATS rather than a thoracotomy.

There are two headline issues: the failure rate of VATS versus thoracotomy, and the extent to which VATS achieves the objectives of less pain and earlier return to work and full comfortable activity. This latter is supported by RCT evidence, the expertise-based comparison, and a consensus of surgeons present at a cardiothoracic meeting.

As far as the failure rate is concerned, the objective is to reduce the recurrence rate from the natural history of $60-70 \%$ to the lowest possible level. In discussion, the audience had seen sufficient instances of recurrence after thoracotomy and pleurectomy (the BTS's 'gold standard') to reject $1 \%$ as a realistic rate, and it is not one that is likely to be proven with any case series of necessary size. More realistically, a reduction in recurrence of pneumothorax from (say) $60 \%$ to $6 \%$ (the overall figure for both methods in $\mathrm{RCTs}^{4}$ ) would be a very good result. With the very low morbidity associated with VATS, a second procedure might well be a very acceptable option for the few patients who experience recurrence.

Primary spontaneous pneumothorax is rarely life threatening. The consensus expressed by the questioned group of over fifty surgeons was that a very effective operation with low morbidity may well be preferable to a possibly better operation with much more severe consequences in terms of pain and other morbidity, particularly when a repeat operation is easily done if pneumothorax recurs.

In drawing conclusions about surgical practice, it is necessary to use all the available evidence, since there is usually insufficient RCT evidence to underpin every aspect of surgical practice. ${ }^{9}$ Bringing all the strands together, there appears to be good reason to advocate VATS in the management of pneumothorax. The evidence base presented, including RCTs, the systematic review and an expertise-based study, all points in that direction. The change in practice documented in the SCTS registry data reflects the acceptance of VATS as the standard of care for pneumothorax. And finally, the opinion gathering exercise completes the triangle.
Competing interests None declared.

Funding None.

Ethical approval Not required.

Guarantor TT.

Acknowledgments I am grateful to the members of the Section of Cardiothoracic Surgery for engaging in the opinion gauging exercise which was made easy by the electronic voting system in the Royal Society of Medicine's lecture theatre. I was unable to obtain consent from the members because publication was not intended at the time. Publication was supported and encouraged by the Section Council, who saw a draft of this manuscript. I am also grateful to the members of the Society for Cardiothoracic Surgery in Great Britain and Ireland who provided the registry data.

\section{REFERENCES}

1 Lewis RJ, Caccavale RJ, Sisler GE. Imaged thoracoscopic surgery: a new thoracic technique for resection of mediastinal cysts. Ann Thorac Surg 1992;53:318-20

2 Lewis RJ, Caccavale RJ, Sisler GE, Mackenzie JW. One hundred consecutive patients undergoing video-assisted thoracic operations. Ann Thorac Surg 1992;54:421-6

3 Henry M, Arnold T, Harvey J. BTS guidelines for the management of spontaneous pneumothorax. Thorax 2003;58(Suppl 2):ii39-ii52

4 Sedrakyan A, van der Meulen J, Lewsey J, Treasure T. Video assisted thoracic surgery for treatment of pneumothorax and lung resections: systematic review of randomised clinical trials. BMJ 2004;329:1008

5 Devereaux PJ, Bhandari M, Clarke M, et al. Need for expertise based randomised controlled trials. BMJ 2005;330:88

6 Ben Nun A, Soudack M, Best LA. Video-assisted thoracoscopic surgery for recurrent spontaneous pneumothorax: the long-term benefit. World $J$ Surg 2006;30:285-90

7 Sedrakyan A, Van Der Meulen J, Lewsey J, Treasure T. Variation in use of video assisted thoracic surgery in the United Kingdom. BMJ 2004;329:1011-2

8 Tan C, Treasure T, Browne J, Utley M, Davies CW, Hemingway H. Seeking consensus by formal methods: a health warning. $J R$ Soc Med 2007; 100:10-4

9 Treasure T. The evidence on which to base practice: different tools for different times. Eur J Cardiothorac Surg 2006;30:819-24 